\title{
The genetic architecture of insecticide resistance within a natural population of Drosophila melanogaster
}

\author{
Takahiro Miyo
}

2-17-4 Misumi-cho, Higashimurayama-shi, Tokyo 189-0023, Japan

Email: takahiro_miyo@hotmail.com

Received 6 January 2012; revised 22 February 2012; accepted 20 March 2012

\begin{abstract}
The dynamics of genetic variation in susceptibility to insecticides within a natural population of Drosophila melanogaster (Meigen) at Katsunuma (Yamanashi Prefecture, Japan) was examined. Two resistance factors for three organophosphate insecticides (OPs), a resistant-type acetylcholinesterase (AChE) and a cytochrome P450 monooxygenase (cytochrome P450), have already been suggested to be involved within the Katsunuma population. In this study, genetic variances were estimated for susceptibility to other classes of chemicals than OPs, permethrin (a pyrethroid) and dichloro-diphenyl-trichloroethane (DDT; an organochlorine), which existed simultaneously with genetic variances for susceptibility to OPs. Analyses of variance for susceptibility to permethrin and DDT showed highly significant variation among isofemale lines from the Katsunuma population, and the genetic variances for susceptibility to each insecticide fluctuated differently during this period. The impacts of fluctuations of genetic variation in susceptibility to one class of insecticides on genetic variation in susceptibility to other classes of insecticides existing simultaneously within the natural population were discussed.
\end{abstract}

Keywords: DDT; Drosophila melanogaster; Genetic Variation; Insecticide Resistance; Permethrin

\section{INTRODUCTION}

Resistance is defined as "the development of an ability in a strain of an organism to tolerate doses of a toxicant which would prove lethal to the majority of individuals in a normal (susceptible) population of the species" [1]. The development of insecticide resistance is a population genetic process within insect pest populations, in which initially rare resistant mutants are selected for by insecticide selection pressures [2]. Because insecticides have been applied to fields for the purpose of reducing pest population densities, insecticides should select for rare resistant mutants as an inevitable consequence. Since insecticide resistance was first documented more than a century ago [1], many pest populations of insects and mites have developed resistance to almost all chemicals [3].

The recent technological progress in molecular genetic approaches has brought about identifications and characterizations of several resistance genes contributing to the main mechanisms of insecticide resistance, including increased detoxification of insecticides and target-site insensitivity to insecticides [4-6]. Increased detoxification of insecticides can be realized by increased activities of metabolic enzymes, which may be caused by many mechanisms, including amplification, over-expression, and point mutations in coding sequences [7]. Increased detoxification has been exemplified by cytochrome P450 monooxygenases (cytochrome P450s), esterases, and glutathion-S-transferases (GSTs) [7]. The other major mechanism of insecticide resistance might involve point mutations that reduce the sensitivity of target molecules to insecticides [8]. Point mutations contributing to targetsite insensitivity have been documented in the voltagegated sodium channel for dichloro-diphenyl-trichloroethane (DDT) and pyrethroids [9], acetylcholinesterase (AChE) for organophosphates (OPs) and carbamates [10], the nicotinic acetylcholine receptor for neonicotinoids and spinosad [11,12], and the $\gamma$-amino butyric acid receptor for cyclodiene insecticides [5].

Katsunuma (Yamanashi Prefecture, Japan) is famous for its wine production, and vineyards extend continuously over this town and surrounding areas. Although Drosophila flies are not recognized as pest species, insecticide selection pressures have been imposed on the Katsunuma population of Drosophila melanogaster (Meigen), owing to management of other pest species [13]. In fact, the Katsunuma population of D. melanogaster exhibited genetic variation in susceptibility to OPs, which was composed of several resistance factors, including a resistant-type AChE and a cytochrome P450 [14,15]. Therefore, it is conceivable that various agents could mold genetic variation within the Katsunuma population, and that the effects of the fluctuations of genetic variation in 
susceptibility to one class of insecticides within the population could also affect those to other classes of insecticides simultaneously.

In this study, genetic variation in susceptibility to other classes of insecticides, a pyrethroid permethrin and an organochlorine DDT, which existed within the Katsunuma population at the same time period as genetic variation in susceptibility to OPs, was estimated. Information on genetic variation in susceptibility to other classes of insecticides as well as OPs would provide further insights into the genetic structures of insecticide resistance within natural populations of insect pests, and these approaches should provide valuable information as a model system for designing appropriate strategies for managing insecticide resistance in other insect pest populations.

\section{MATERIALS AND METHODS}

Fluctuations of genetic variation in susceptibility to a pyrethroid permethrin and an organochlorine DDT within the Katsunuma population were investigated. Mortality data of isofemale lines published in [16] were used to estimate genetic variances in susceptibility to permethrin and DDT within the Katsunuma population. Isofemale lines were established from the natural population of $D$. melanogaster collected at Katsunuma (Yamanashi Prefecture, Japan) in summer and fall, 1997 and 1998. Detailed information on the isofemale lines and rearing conditions was described in [16].

By adopting the filter-paper-contact method, the susceptibility of each isofemale line was evaluated as mortality under one concentration of each insecticide. Based on $\mathrm{LC}_{50}$ values (insecticide concentrations that kill 50\% of the tested population) of a standard laboratory strain Canton-S, the concentrations were determined as 1.25 $\mathrm{mg}$ permethrin $/ \mathrm{cm}^{2}$ and $0.125 \mathrm{mg} \mathrm{DDT} / \mathrm{cm}^{2}$, respectively. The origins and purity of the chemicals and bioassay conditions were described in [16].

Variance components, a between-line component $\left(V_{b}\right)$ and a within-line component $\left(V_{w}\right)$, were estimated, following the analysis of variance $[14,17]$. Mortality data of isofemale lines were arcsine-square-root transformed, prior to the analyses. The analyses of variance were conducted using mortality data without adjustment by control mortality, because control mortality of each line was reasonably small: $0.032 \pm 0.0075$ (1997 summer), 0.028 \pm 0.0038 (1997 fall), $0.014 \pm 0.0045$ (1998 summer), and $0.018 \pm 0.0028$ (1998 fall) (mean \pm SE over isofemale lines within each population). In fact, Abbott's adjustment using control mortality [18] provided essentially the same results (data not shown).

Genetic variance $\left(V_{g}\right)$ in susceptibility to each insecticide within each population was estimated, after correction was made using $V_{w}[14,19]$. The number of replications was usually three, but not always so; therefore, the harmonic mean of replication numbers was used for calculating variance components [17]. Standard errors of variance components were estimated approximately, based on sampling variances of mean squares [20].

\section{RESULTS AND DISCUSSION}

Mortality of each isofemale line after 24 hours was examined at $1.25 \mathrm{mg} / \mathrm{cm}^{2}$ permethrin and at $0.125 \mathrm{mg} / \mathrm{cm}^{2}$ DDT [16]. The results of the analyses of variance for susceptibility to permethrin and DDT were listed in Table 1. In each season, there was significant variation between isofemale lines in susceptibility to each insecticide $\left(P<10^{-5}\right)$. The concentrations adopted for bioassays were 3 and 49 times higher than the $L_{50}$ values of the laboratory strain Canton-S for the respective insecticides. Even under the higher insecticide concentrations than $\mathrm{LC}_{50}$ values of Canton-S, which is usually used for a susceptible wild-type strain, the Katsunuma population of $D$. melanogaster showed significant genetic variances in susceptibility to permethrin (a pyrethroid) and DDT (an organochlorine) (Figure 1), at a time when this population exhibited significant genetic variances for susceptibility to the three OPs [14]. However, the patterns of the fluctuations were different between genetic variances in susceptibility to the two insecticides. Genetic variances of permethrin susceptibility were $0.070 \pm 0.0162$ (estimate \pm SE) (1997 summer), $0.045 \pm 0.0063$ (1997 fall), $0.047 \pm 0.0152$ (1998 summer), and $0.030 \pm 0.0085$ (1998 fall), and did not fluctuate significantly during the two years (Figure 1), while the mean susceptibility level for permethrin did not change significantly [16]. On the other hand, genetic variances of DDT susceptibility were $0.075 \pm 0.0153$ (1997 summer), $0.065 \pm 0.0081$ (1997 fall), $0.019 \pm 0.0111$ (1998 summer), and $0.088 \pm 0.0166$ (1998 fall), and did not change significantly in 1997 but increased significantly in 1998 (Figure 1), which was the same fluctuation pattern as for susceptibility to the three OPs [14] although the mean susceptibility to DDT tended to decrease with time [16]. Within-line variances were $0.042 \pm 0.0049$ (estimate \pm SE) (1997 summer), $0.039 \pm$ 0.0023 (1997 fall), $0.028 \pm 0.0044$ (1998 summer), and $0.040 \pm 0.0041$ (1998 fall) for susceptibility to permethrin, whereas within-line variances for susceptibility to DDT were $0.027 \pm 0.0031$ (1997 summer), $0.039 \pm 0.0024$ (1997 fall), $0.042 \pm 0.0065$ (1998 summer), and $0.036 \pm$ 0.0037 (1998 fall). Because within-line variances were almost the same among the seasons, which were also the same magnitude as those for susceptibility to the three OPs [14], it can be assumed that genetic variances based on experiments conducted under reasonably controlled conditions.

Generally speaking, DDT and pyrethroid insecticides act on the same target site, the voltage-gated sodium channel [21]; in addition, many specific genes for detoxification 
Table 1. Analysis of variance for susceptibility to permethrin and DDT within the Katsunuma population of D. melanogaster.

\begin{tabular}{|c|c|c|c|c|c|c|c|c|c|c|}
\hline \multirow{2}{*}{$\begin{array}{c}\text { Chemicals } \\
\text { source }\end{array}$} & \multicolumn{5}{|c|}{ Permethrin } & \multicolumn{5}{|c|}{ DDT } \\
\hline & $\mathrm{d} f$ & $M S$ & $F$ & $P$ & Expected $M S$ & $\mathrm{~d} f$ & MS & $F$ & $P$ & Expected $M S$ \\
\hline $\begin{array}{l}\text { (a) } 1997 \text { summer } \\
\text { Between lines }\end{array}$ & 75 & 0.2937 & 6.92 & $<10^{-23}$ & $\sigma_{\mathrm{w}}^{2}+3.00 \sigma_{\mathrm{B}}^{2}$ & 75 & 0.2794 & 10.18 & $<10^{-32}$ & $\sigma_{\mathrm{w}}^{2}+3.00 \sigma_{\mathrm{B}}^{2}$ \\
\hline Within lines & 152 & 0.0425 & & & $\sigma_{\mathrm{w}}^{2}$ & 152 & 0.0274 & & & $\sigma_{\mathrm{w}}^{2}$ \\
\hline $\begin{array}{l}\text { (b) } 1997 \text { fall } \\
\text { Between lines }\end{array}$ & 259 & 0.2161 & 5.55 & $<10^{-63}$ & $\sigma_{\mathrm{w}}^{2}+3.06 \sigma_{\mathrm{B}}^{2}$ & 256 & 0.2765 & 7.07 & $<10^{-80}$ & $\sigma_{\mathrm{w}}^{2}+3.06 \sigma_{\mathrm{B}}^{2}$ \\
\hline Within lines & 556 & 0.0389 & & & $\sigma_{\mathrm{w}}^{2}$ & 547 & 0.0391 & & & $\sigma_{\mathrm{w}}^{2}$ \\
\hline $\begin{array}{l}\text { (c) } 1998 \text { summer } \\
\text { Between lines }\end{array}$ & 38 & 0.1998 & 7.14 & $<10^{-13}$ & $\sigma_{\mathrm{w}}^{2}+3.04 \sigma_{\mathrm{B}}^{2}$ & 38 & 0.1408 & 3.39 & $<10^{-5}$ & $\sigma_{\mathrm{w}}^{2}+3.04 \sigma_{\mathrm{B}}^{2}$ \\
\hline Within lines & 81 & 0.0280 & & & $\sigma_{\mathrm{w}}^{2}$ & 81 & 0.0416 & & & $\sigma_{\mathrm{w}}^{2}$ \\
\hline $\begin{array}{l}\text { (d) } 1998 \text { fall } \\
\text { Between lines }\end{array}$ & 93 & 0.1697 & 4.23 & $<10^{-16}$ & $\sigma_{\mathrm{w}}^{2}+3.00 \sigma_{\mathrm{B}}^{2}$ & 93 & 0.3370 & 9.32 & $<10^{-37}$ & $\sigma_{\mathrm{w}}^{2}+3.00 \sigma_{\mathrm{B}}^{2}$ \\
\hline Within lines & 188 & 0.0401 & & & $\sigma_{\mathrm{w}}^{2}$ & 188 & 0.0362 & & & $\sigma_{\mathrm{w}}^{2}$ \\
\hline
\end{tabular}

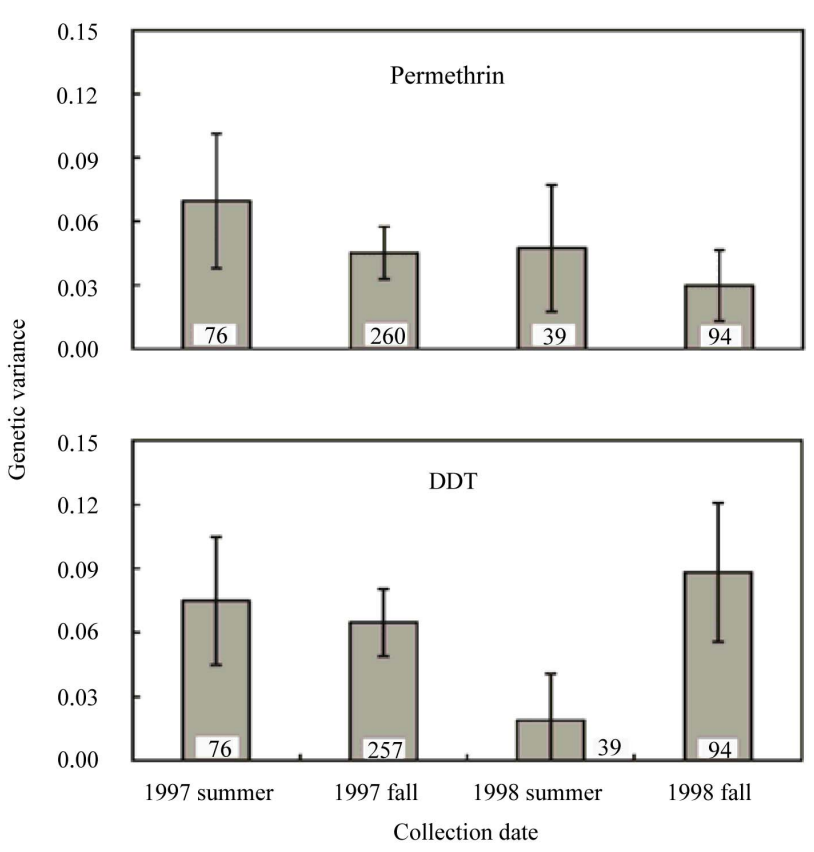

Figure 1. Seasonal fluctuations in genetic variances of susceptibility to permethrin and DDT within the Katsunuma population of $D$. melanogaster. Numbers in the graphs represent the numbers of isofemale lines examined for estimation. Error bars indicate $95 \%$ confidence intervals.

enzymes, including cytochrome P450s and GSTs, have been identified for resistance to DDT and/or pyrethroids $[6,7,22]$. Therefore, it might be presumed that a single resistance factor within the Katsunuma population could confer cross-resistance to both insecticides. However, this hypothesis cannot explain the different patterns observed in both the fluctuations of the genetic variances (Figure 1) and the mean levels [16] between susceptibility to the two insecticides. Although there remain many aspects to be revealed in the fluctuations of genetic variation in resistance to DDT and permethrin within the natural population, the most probable explanation for the differences in fluctuation patterns is that the Katsunuma population possessed several resistance factors for DDT and permethrin, in which different genes might have main effects on resistance to the respective insecticides. If the identical gene had large effects on resistance to the two chemicals, the levels and genetic variances would have shown the same fluctuation patterns.

In the course of studies on genetic variation in resistance to OPs within the Katsunuma population, we have identified two resistance factors for OPs, one on chromosome 2 ( $\sim$ II-62) and the other on chromosome 3 ( III-50) [23]. The resistance factor mapped at $\sim \mathrm{II}-62$ on chromosome 2 was recently suggested to be a cytochrome P450, based on in vivo biochemical assays [15]. Other laboratories have provided the possibility that one or some cytochrome P450 (Cyp) genes around this map position might be involved in resistance to several insecticides including DDT $[24,25]$. Especially, Cyp6g1 has received very much attention and been suggested to be involved in resistance to DDT (an organochlorine) and imidacloprid (a neonicotinoid insecticide) $[24,26]$ and diazinon (an OP) [27]. Because the population was different, the Katsunuma population had not necessarily developed the same monooxygenase-based resistance mechanisms as other populations [28]. Although we have no idea of whether or not the Cyp gene for OP resistance could contribute to resistance to the two chemicals within the Katsunuma population, genetic variation in susceptibility to DDT and permethrin cannot be explained by only this Cyp gene, even though this gene could have shown cross-resistance to the two insecticides.

This study demonstrated the significant genetic variances in susceptibility to DDT maintained within the Katsunuma population, which fluctuated among seasons, even after a long period of time since its registration had been expired (Figure 1). It had also been demonstrated that the mean level of susceptibility to DDT fluctuated among seasons [16]. Because it is hard to consider that 
the fluctuations were caused by direct application of DDT during those days, the fluctuations could be attributable to other insecticides, including OPs and pyrethroids, applied for controlling other insect pests for graperies [16]. Although the resistant-type AChE, which had the large effects on resistance to OPs within the Katsunuma population [29], actually does not participate directly in resistance to DDT, it could possibly influence genetic variation in DDT resistance via correlations caused by multiple-resistance. In fact, significant but temporal correlations between susceptibility to DDT and susceptibility to OPs and permethrin were observed within the Katsunuma population [16]. In addition, it is interesting whether or not the Cyp gene identified for OP resistance could contribute to genetic variation in susceptibility to DDT and permethrin via cross-resistance.

We observed for two years that the susceptibility to the three OPs tended to increase when the Katsunuma population increased in the fall; however, in case of DDT and permethrin, a tendency towards an increase in susceptibility was not observed when the population increased [16]. Therefore, it was suggested that, unlike resistance to the three OPs, the factors for DDT and permethrin resistance might not entail fitness costs under the density-independent condition, which the Katsunuma population encountered in the fall. However, fitness costs were suggested for pyrethroid resistance in the house fly [30] and in the mosquito (Culex pipiens quinquefasciatus) [31], whereas a fitness advantage of DDT resistance was suggested in D. melanogaster [32]. Because fitness costs were dependent on ecological and environmental conditions under which fitness components were measured $[33,34]$, it is possible that the factors for DDT and permethrin resistance within the Katsunuma population may exhibit fitness costs under different conditions.

Genetic variation in susceptibility to DDT within the Katsunuma population was suggested to be influenced by the resistance factors for OPs and pyrethroids. Although it seems likely that the dynamics of genetic variation in susceptibility within natural populations of insect pests is very complex, it is expected that detailed research in the future on genetic variation in susceptibility to insecticides within natural populations of $D$. melanogaster would provide further insights into resistance management strategies as a model system.

\section{ACKNOWLEDGEMENTS}

I would like to express my sincere appreciation to Emeritus Professor Yuzuru Oguma at the University of Tsukuba, Japan and Professor Brian Charlesworth at the University of Edinburgh, UK for continuous support for my study and critical reading of the manuscript.

\section{REFERENCES}

[1] Mullin, C.A. and Scott, J.G. (1992) Biomolecular basis for insecticide resistance: Classification and comparisons. In: Mullin, C.A. and Scott, J.G. Eds., Molecular Mechanisms of Insecticide Resistance, American Chemical Society, Washington DC, 1-13.

[2] Crow, J.F. (1957) Genetics of insect resistance to chemicals. Annual Review of Entomology, 2, 227-246. doi:10.1146/annurev.en.02.010157.001303

[3] Roush, R.T. and McKenzie, J.A. (1987) Ecological genetics of insecticide and acaricide resistance. Annual Review of Entomology, 32, 361-380. doi:10.1146/annurev.en.32.010187.002045

[4] Oakeshott, J.G., Horne, I., Sutherland, T.D. and Russell, R.J. (2003) The genomics of insecticide resistance. Genome Biology, 4, 202. doi:10.1186/gb-2003-4-1-202

[5] French-Constant, R.H., Daborn, P.J. and Le Goff, G. (2004) The genetics and genomics of insecticide resistance. Trends in Genetics, 20, 163-170. doi:10.1016/j.tig.2004.01.003

[6] Hemingway, J., Hawkes, N.J., McCarroll, L. and Ranson, $\mathrm{H}$. (2004) The molecular basis of insecticide resistance in mosquitoes. Insect Biochemistry and Molecular Biology, 34, 653-665. doi:10.1016/j.ibmb.2004.03.018

[7] Li, X., Schuler, M.A. and Berenbaum, M.R. (2007) Molecular mechanisms of metabolic resistance to synthetic and natural xenobiotics. Annual Review of Entomology, 52, 231-253. doi:10.1146/annurev.ento.51.110104.151104

[8] Morton, R.A. (1993) Evolution of Drosophila insecticide resistance. Genome, 36, 1-7. doi:10.1139/g93-001

[9] Usherwood, P.N.R., Davies, T.G.E., Mellor, I.R., O’Reilly, A.O., Peng, F., Vais, H., Khambay, B.P.S., Field, L.M. and Williamson, M.S. (2007) Mutations in DIIS5 and the DIIS4-S5 linker of Drosophila melanogaster sodium channel define binding domains for pyrethroids and DDT. FEBS Letters, 581, 5485-5492. doi:10.1016/j.febslet.2007.10.057

[10] Fournier, D. (2005) Mutations of acetylcholinesterase which confer insecticide resistance in insect populations. Chemico-Biological Interactions, 157-158, 257-261. doi:10.1016/j.cbi.2005.10.040

[11] Perry, T., McKenzie, J.A. and Batterham, P. (2007) A $D \propto 6$ knockout strain of Drosophila melanogaster confers a high level of resistance to spinosad. Insect Biochemistry and Molecular Biology, 37, 184-188. doi:10.1016/j.ibmb.2006.11.009

[12] Perry, T., Heckel, D.G., McKenzie, J.A. and Batterham, P. (2008) Mutations in $D \alpha 1$ or $D \beta 2$ nicotinic acetylcholine receptor subunits can confer resistance to neonicotinoids in Drosophila melanogaster. Insect Biochemistry and Molecular Biology, 38, 520-528. doi:10.1016/j.ibmb.2007.12.007

[13] Miyo, T., Takamori, H., Kono, Y. and Oguma, Y. (2001) Genetic variation and correlations among responses to five insecticides within natural populations of Drosophila melanogaster (Diptera: Drosophilidae). Journal of Economic Entomology, 94, 223-232. doi:10.1603/0022-0493-94.1.223

[14] Miyo, T., Oguma, Y. and Charlesworth, B. (2006) Sea- 
sonal fluctuation in susceptibility to insecticides within natural populations of Drosophila melanogaster. II. Features of genetic variation in susceptibility to organophosphate insecticides within natural populations of $D$. melanogaster. Genes \& Genetic Systems, 81, 273-285. doi:10.1266/ggs.81.273

[15] Miyo, T. and Oguma, Y. (2010) Contributions of threesite mutations in acetylcholinesterase and cytochrome P450 to genetic variation in susceptibility to organophosphate insecticides within a natural population of Drosophila melanogaster. Population Ecology, 52, 159-169. doi:10.1007/s10144-009-0157-1

[16] Miyo, T., Akai, S. and Oguma, Y. (2000) Seasonal fluctuation in susceptibility to insecticides within natural populations of Drosophila melanogaster: Empirical observations of fitness costs of insecticide resistance. Genes \& Genetic Systems, 75, 97-104. doi:10.1266/ggs.75.97

[17] Mukai, T. (1964) The genetic structure of natural populations of Drosophila melanogaster. I. Spontaneous mutation rate of polygenes controlling viability. Genetics, 50, 1-19.

[18] Abbott, W.S. (1925) A method of computing the effectiveness of an insecticide. Journal of Economic Entomology, 18, 265-267.

[19] David, J.R., Gibert, P., Legout, H., Pétavy, G., Capy, P. and Moreteau, B. (2005) Isofemale lines in Drosophila: An empirical approach to quantitative trait analysis in natural populations. Heredity, 94, 3-12. doi:10.1038/sj.hdy.6800562

[20] Takano, T., Kusakabe, S. and Mukai, T. (1987) The genetic structure of natural populations of Drosophila melanogaster. $\mathrm{XX}$. Comparison of genotype-environment interaction in viability between a northern and a southern population. Genetics, 117, 245-254.

[21] Davies, T.G.E., Field, L.M., Usherwood, P.N.R. and Williamson, M.S. (2007) DDT, pyrethrins, pyrethroids and insect sodium channels. IUBMB Life, 59, 151-162. doi:10.1080/15216540701352042

[22] Scott, J.G. (1999) Cytochromes P450 and insecticide resistance. Insect Biochemistry and Molecular Biology, 29, 757-777. doi:10.1016/S0965-1748(99)00038-7

[23] Miyo, T., Kono, Y. and Oguma, Y. (2002) Genetic basis of cross-resistance to three organophosphate insecticides in Drosophila melanogaster (Diptera: Drosophilidae). Journal of Economic Entomology, 95, 871-877. doi:10.1603/0022-0493-95.5.871

[24] Daborn, P.J., Yen, J.L., Bogwitz, M.R., Le Goff, G., Feil, E., Jeffers, S., Tijet, N., Perry, T., Heckel, D., Batterham, P., Feyereisen, R., Wilson, T.G. and French-Constant, R.H. (2002) A single P450 allele associated with insecticide resistance in Drosophila. Science, 297, 2253-2256.

\section{doi:10.1126/science.1074170}

[25] Festucci-Buselli, R.A., Carvalho-Dias, A.S., de OliveiraAndrade, M., Caixeta-Nunes, C., Li, H.-M., Stuart, J.J., Muir, W., Scharf, M.E. and Pittendrigh, B.R. (2005) Expression of Cyp6g1 and Cyp12d1 in DDT resistant and susceptible strains of Drosophila melanogaster. Insect Molecular Biology, 14, 69-77. doi:10.1111/j.1365-2583.2005.00532.x

[26] Joußen, N., Heckel, D.G., Haas, M., Schuphan, I. and Schmidt, B. (2008) Metabolism of imidacloprid and DDT by P450 CYP6G1 expressed in cell cultures of Nicotiana tabacum suggests detoxification of these insecticides in Cyp6g1-overexpressing strains of Drosophila melanogaster, leading to resistance. Pest Management Science, 64, 6573.

[27] Pyke, F.M., Bogwitz, M.R., Perry, T., Monk, A., Batterham, P. and McKenzie, J.A. (2004) The genetic basis of resistance to diazinon in natural populations of Drosophila melanogaster. Genetica, 121, 13-24. doi:10.1023/B:GENE.0000019920.71944.2b

[28] Scott, J.G. and Kasai, S. (2004) Evolutionary plasticity of monooxygenase-mediated resistance. Pesticide Biochemistry and Physiology, 78, 171-178. doi:10.1016/j.pestbp.2004.01.002

[29] Miyo, T. (2011) Population model of fluctuations in organophosphate resistance of Drosophila melanogaster: Roles of a mutated acetylcholinesterase and a cytochrome P450. Russian Journal of Ecology, 42, 510-517. doi:10.1134/S1067413611060178

[30] Rinkevich, F.D., Hamm, R.L., Geden, C.J. and Scott, J.G. (2007) Dynamics of insecticide resistance alleles in house fly populations from New York and Florida. Insect Biochemistry and Molecular Biology, 37, 550-558. doi:10.1016/j.ibmb.2007.02.013

[31] Hardstone, M.C., Lazzaro, B.P. and Scott, J.G. (2009) The effect of three environmental conditions on the fitness of cytochrome P450 monooxygenase-mediated permethrin resistance in Culex pipiens quinquefasciatus. BMC Evolutionary Biology, 9, 42. doi:10.1186/1471-2148-9-42

[32] McCart, C. and ffrench-Constant, R.H. (2008) Dissecting the insecticide-resistance-associated cytochrome P450 gene Cyp6g1. Pest Management Science, 64, 639-645. doi:10.1002/ps.1567

[33] Coustau, C., Chevillon, C. and ffrench-Constant, R. (2000) Resistance to xenobiotics and parasites: Can we count the cost? Trends in Ecology and Evolution, 15, 378-383. doi:10.1016/S0169-5347(00)01929-7

[34] Gassmann, A.J., Carrière, Y. and Tabashnik, B.E. (2009) Fitness costs of insect resistance to Bacillus thuringiensis. Annual Review of Entomology, 54, 147-163. doi:10.1146/annurev.ento.54.110807.090518 